\title{
Avaliação morfológica da união entre adesivo/resina composta e dentina irradiada com laser Er:YAG e laser Nd:YAG: estudo comparativo por microscopia de varredura
}

\author{
Morphological evaluation of the bonding between \\ adhesive/composite resin and dentin irradiated with Er:YAG and \\ Nd:YAG lasers: comparative study using scanning microscopy
}

\author{
Margareth ODA* \\ Denise Cerqueira OLIVEIRA** \\ Edson Aparecido LIBERTI***
}

\begin{abstract}
ODA, M.; OLIVEIRA, D. C.; LIBERTI, E. A. Avaliação morfológica da união entre adesivo/resina composta e dentina irradiada com laser Er:YAG e laser Nd:YAG: estudo comparativo por microscopia de varredura. Pesqui Odontol Bras, v. 15, n. 4, p. 283-289, out./dez. 2001.
\end{abstract}

\begin{abstract}
Desde a introdução de sistemas adesivos nos procedimentos restauradores com materiais estéticos, tem-se estudado de forma abrangente o tipo de tratamento na superfície dentinária para se consagrar uma técnica ideal. A utilização do ácido fosfórico a $37 \%$ sobre a dentina é atualmente ainda o método que mais se domina em termos de adesividade. Porém, métodos de tratamento alternativos, na dentina, têm sido discutidos na literatura, entre eles a aplicação de alguns tipos de irradiação a laser. O objetivo desta pesquisa foi avaliar morfologicamente a união adesivo/resina composta e dentina irradiada com laser Er:YAG e laser Nd:YAG num estudo comparativo por microscopia de varredura, utilizados em substituição ao condicionamento ácido ou associados ao mesmo. Foram utilizados dentes bovinos recém-extraídos, que receberam um preparo de cavidade de classe V, e restauração com sistema adesivo e resina composta fotoativada. Como variantes alguns deles receberam o tratamento prévio de irradiação com laser Er:YAG e outros de irradiação com laser Nd:YAG, à aplicação do agente adesivo e da resina composta. As amostras foram seccionadas, preparadas para microscopia de varredura e submetidas a uma análise morfológica, e foram feitos os registros por meio de fotomicrografias. Com base nessas observações microscópicas, concluímos que somente no tratamento da superficie da dentina com laser Er:YAG e condicionamento ácido houve penetração da resina na dentina. Nos tratamentos com laser Nd:YAG, houve apenas aparente superposição da resina na superficie dentinária, sugerindo que houve oclusão dos túbulos com características de fusão na dentina superficial.
\end{abstract}

UNITERMOS: Lasers; Resinas compostas.

\section{INTRODUÇÃO}

Adesividade é um assunto muito discutido desde que BUONOCORE ${ }^{2}$, em 1955, preconizou o condicionamento ácido. Inicialmente, este procedimento era de indicação exclusiva para o esmalte, e utilizado como tratamento químico para favorecer o vedamento marginal e melhorar a adesão das restaurações com resina composta.

Devido ao fato de a dentina possuir como propriedades de sua estrutura, umidade e permeabilidade, há uma constante preocupação em se desen- volver materiais ou métodos que sejam compativeis a esta superficie favorecendo o devido selamento nas restaurações. O condicionamento ácido na dentina muitas vezes expõe os túbulos dentinários aumentando a permeabilidade e conseqüente infiltração bacteriana ou passagem de fluidos e estímulos.

Atualmente, um dos passos operatórios alternativos para o tratamento dentinário é o uso da irradiação com laser Er:YAG ${ }^{4}$, que vem sendo estudado não somente para a realização de preparo cavitário, remoção de tecido cariado como no condicionamento de esmalte e dentina. Outro tipo de laser que tam-

\footnotetext{
* Professora Doutora do Departamento de Dentística da Faculdade de Odontologia da USP.

** Professora Adjunta do Departamento de Saúde da Universidade Estadual de Feira de Santana.

*** Professor Associado do Departamento de Anatomia do Instituto de Ciências Biomédicas da USP.
} 
ODA, M.; OLIVEIRA, D. C.; LIBERTI, E. A. Avaliação morfológica da união entre adesivo/resina composta e dentina irradiada com laser Er:YAG e laser Nd:YAG: estudo comparativo por microscopia de varredura. Pesqui Odontol Bras, v. 15, n. 4, p. 283-289, out./dez. 2001.

bém vem sendo pesquisado é o Nd:YAG, principalmente seu efeito em esmalte e dentina ${ }^{3,6,7,8,16,17,19,24,25}$.

Os autores WIGDOR et al. ${ }^{26}(1992)$ avaliaram por microscopia eletrônica de varredura (MEV) a dentina irradiada por alguns lasers. O que observaram do efeito do Nd:YAG foi fusão da dentina intertubular e a variação de diâmetro dos túbulos dentinários, confirmando o observado por DEDERICH et $a l^{5}$ em 1984, que salientam ainda uma recristalização da dentina; para o de Er:YAG, o aspecto foi bem diferente, com uma boa definição de túbulos dentinários e ausência de "debris".

A idéia para esta pesquisa surgiu devido ao fato de estarmos na era da Odontologia adesiva e principalmente em um momento de extrema valorização da estética, havendo cada vez mais necessidade de procedimentos que favoreçam melhor união entre estrutura dentária e materiais restauradores estéticos. Preocupados com este assunto, TANJI et al. ${ }^{19}$, em 1998, observaram por microscopia de varredura as alterações de superficie dentinária de canais radiculares irradiados com lasers Er:YAG e Nd:YAG. Concluíram que com o laser Nd:YAG ocorreu a fusão e a recristalização da dentina, obliterando alguns túbulos dentinários e que com o laser Er:YAG alguns canalículos dentinários foram abertos pelo efeito de ablação.

Mesmo resultado foi encontrado por MATSUMO$\mathrm{TO}^{13}$, que em 1998, ao estudar as mudanças morfológicas de dentes tratados com o laser Er:YAG, observou por $\mathrm{MEV}$, que a superficie dentinária apresentava-se com túbulos abertos.

Em 1999, YONAGA et al. ${ }^{23}$ avaliaram em MEV, os efeitos do laser Nd:YAG em dentina cervical, e observaram que após irradiação com $2 \mathrm{~W}, 100 \mathrm{~mJ}$, $20 \mathrm{~Hz}$, a dentina se apresentava com a maioria dos túbulos ocluídos ou estreitados, com características de fusão na dentina superficial.

Neste mesmo ano, KAWABATA et al. ${ }^{12}$ estudaram morfologicamente os efeitos de diversos tipos de laser na dentina e concluíram que o laser Nd:YAG selou efetivamente os túbulos dentinários, sem carbonização ou fratura, e que com o laser Er:YAG a superficie mostrava-se irregular, com túbulos abertos.

A proposta desta pesquisa experimental, in vitro, é de rever na literatura o emprego do laser Er:YAG e Nd:YAG em Dentística Restauradora, como tratamento operatório prévio ao procedimento adesivo e restaurador e avaliar morfologicamente a união adesivo/resina composta e dentina tratada com irradiação laser Er:YAG e com laser Nd:YAG por meio de um estudo comparativo por microscopia de varredura.

\section{MATERIAL E MÉTODOS}

Para esta pesquisa, foram utilizados dentes naturais recém-extraídos de bovinos. Os elementos dentais foram submetidos a uma profilaxia e armazenados em soro fisiológico 0,9\% (Aster Produtos Médicos Ltda.) até o início dos trabalhos, para se manterem hidratados.

\section{Preparo dos dentes}

Foram preparadas cavidades de classe V, na superficie vestibular dos dentes ${ }^{1}$. As dimensões dos preparos foram de aproximadamente 4 milimetros de altura, 6 de largura e 3 de profundidade. Os preparos foram realizados com ponta diamantada cilíndrica, número 1092 (KG Sorensen) em alta rotação e total refrigeração.

\section{Tratamento da superfície dentinária e procedimento adesivo}

Os dentes foram divididos em grupos possibilitando a avaliação de diversos aspectos por meio da microscopia de varredura. No $1^{\circ}$ grupo, os preparos receberam um tratamento na superficie da dentina com laser Er:YAG, seguidos do procedimento adesivo e restaurador com resina composta fotoativada, como recomendado pelo fabricante. O material restaurador selecionado para todos os grupos foi o Single Bond - frasco único - 3M e resina composta Z100 (3M Dental Products).

No $2^{\circ}$ Grupo, o tratamento na superficie da dentina foi realizado com laser Nd:YAG, seguido do procedimento adesivo e restaurador.

No $3^{\circ}$ Grupo, o tratamento na superficie dentinária foi realizado com laser Er:YAG, seguido do procedimento adesivo e restauração com resina composta. Neste grupo, não foi aplicado o condicionamento ácido.

No $4^{\circ}$ Grupo, o tratamento na superficie da dentina foi realizado com laser Nd:YAG, e seguido do procedimento adesivo e restauração. Neste grupo, também não foi aplicado o condicionamento ácido.

Neste trabalho, utilizamos o laser Nd:YAG Pulse Master 1000 (American Dental Technology, Southfield, MI, EUA), pertencente ao Laboratório Experimental de Laser em Odontologia (LELO), da Faculdade de Odontologia, da Universidade de São Paulo. Este equipamento que possui comprimento de onda de $1.064 \mu \mathrm{m}$, foi utilizado com os parâmetros $0,6 \mathrm{~W}, 15 \mathrm{~Hz}, 40 \mathrm{~mJ}$, com a fibra óptica de $320 \mu \mathrm{m}$, sendo utilizada em varredura por toda superfície dentinária. O tempo de irradiação foi de 30 segundos. A distância da ponta da fibra até a denti- 
ODA, M.; OLIVEIRA, D. C.; LIBERTI, E. A. Avaliação morfológica da união entre adesivo/resina composta e dentina irradiada com laser Er:YAG e laser Nd:YAG: estudo comparativo por microscopia de varredura. Pesqui Odontol Bras, v. 15, n. 4, p. 283-289, out./dez. 2001.

na foi de aproximadamente $1 \mathrm{~mm}$. Durante a irradiação, o preparo cavitário foi refrigerado com jatos de ar da seringa triplice.

O aparelho de laser Er:YAG utilizado foi o modelo KaVo Key Laser (KaVo Co, Alemanha), com comprimento de onda de $2,94 \mu \mathrm{m}$, bem absorvido pela água e hidroxiapatita. Possui ainda energia ajustável de 60 a 500 mJ, taxa de repetição de 1 a $15 \mathrm{~Hz}$ e duração de pulso de 250 a $500 \mu$ s. Neste trabalho, foram utilizados os parâmetros de $2 \mathrm{~Hz}, 80 \mathrm{~mJ}$ focado, durante 30 segundos, em varredura. Este laser trabalha na forma não contato, e o foco ideal é estabelecido entre 12 e $15 \mathrm{~mm}$ da lente de saída até o tecido irradiado. A refrigeração deste aparelho ocorre através de um spray de água, o qual irá atuar no processo de ablação.

Tanto o aparelho de laser Nd:YAG como o de laser Er:YAG foram utilizados de acordo com as instruções dos fabricantes, observando-se todas as normas de segurança preconizadas pela Academy of Laser Dentistry.

Após esses procedimentos, todos os elementos dentais foram seccionados no sentido longitudinal, com auxílio de cinzel e martelo, no centro da restauração e foram preparados os corpos-de-prova no tamanho adequado para a leitura por microscopia

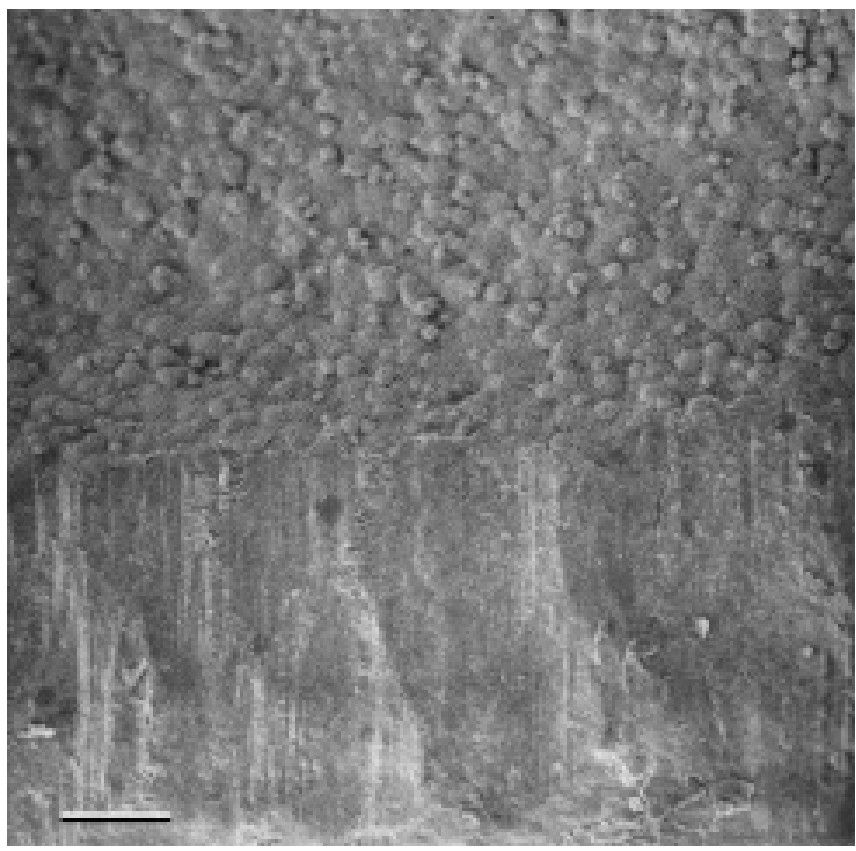

FIGURA 1 - Micrografia de varredura da junção resina/dentina tratada com laser Er:YAG e prévia aplicação de ácido fosfórico a 37\%. Nota-se que os túbulos estão cortados e que houve penetração da resina (barra de calibração $=10 \mu \mathrm{m})$. de varredura. Foi selecionado o melhor ponto na junção dente/restauração. Os corpos-de-prova foram submetidos ao procedimento de secagem em estufa a $37^{\circ} \mathrm{C}$, por 12 horas e montados em "stubs". As amostras foram metalizadas com ouro em um aparelho Balzers SCD-040, e analisadas em um microscópio eletrônico de varredura JEOL 6100, do setor de Microscopia Eletrônica do Departamento de Histologia e Embriologia do Instituto de Ciências Biomédicas da Universidade de São Paulo.

\section{RESULTADOS}

Nos espécimes do $1^{\circ}$ grupo, que receberam tratamento na superficie da dentina com laser Er:YAG e procedimento adesivo e restaurador com resina composta, observou-se que os túbulos dentinários estão cortados e que aparentemente houve penetração da resina na dentina (Figura 1).

Nos espécimes do $2^{\circ}$ grupo, em que a superficie da dentina recebeu o tratamento com laser Nd:YAG e procedimento adesivo e restaurador com resina composta, foi possivel observar a linha de união da dentina com a resina não havendo abertura de túbulos dentinários, sugerindo que os túbulos estavam vedados (Figura 2 ).

Com relação aos espécimes do $3^{\circ}$ grupo, cujo

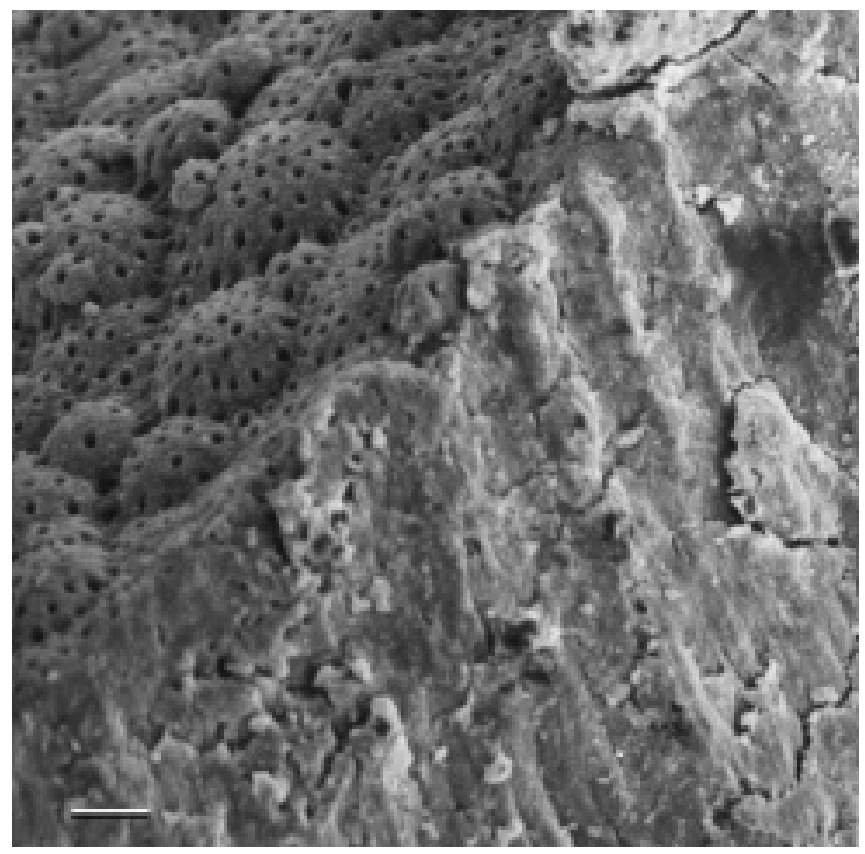

FIGURA 2 - Micrografia de varredura da junção resina/dentina tratada com laser Nd:YAG. Observa-se a não-abertura de túbulos (barra de calibração $=100 \mu \mathrm{m}$ ). 
ODA, M.; OLIVEIRA, D. C.; LIBERTI, E. A. Avaliação morfológica da união entre adesivo/resina composta e dentina irradiada com laser Er:YAG e laser Nd:YAG: estudo comparativo por microscopia de varredura. Pesqui Odontol Bras, v. 15, n. 4, p. 283-289, out./dez. 2001.

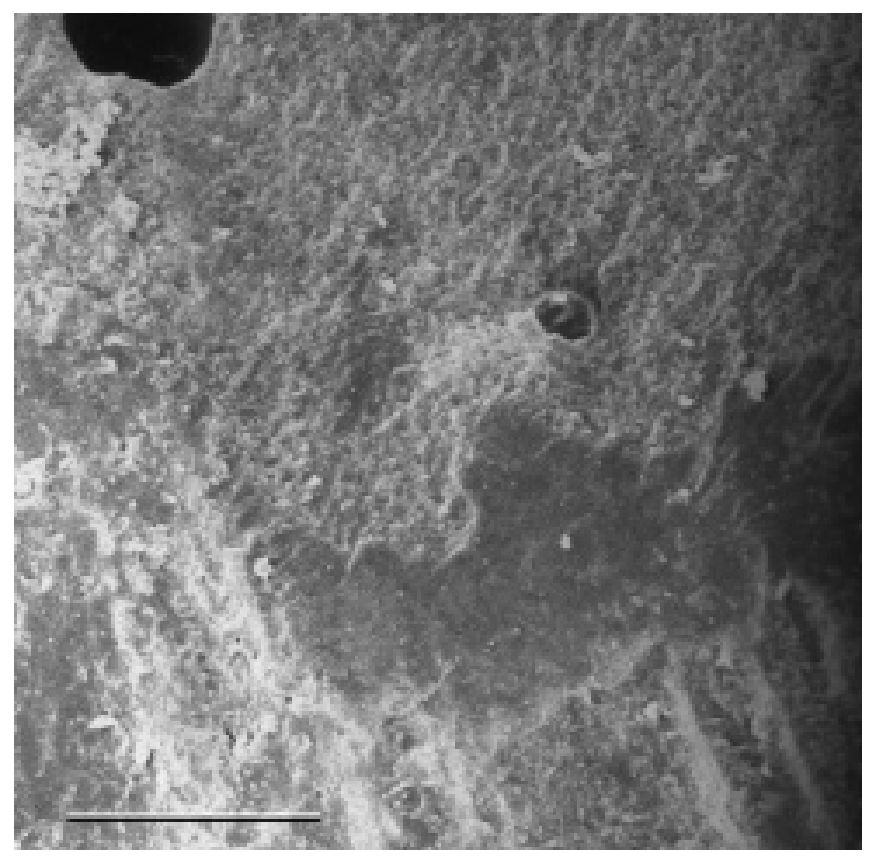

FIGURA 3 - Micrografia de varredura da junção sistema adesivo/resina/dentina tratada com laser Er:YAG. Nesta vista, a resina encontra-se espalhada, sem penetração nos túbulos (barra de calibração $=50 \mu \mathrm{m}$ ).

tratamento na superficie da dentina foi realizado com laser Er:YAG e procedimento adesivo e restaurador com resina composta, sem a aplicação do condicionamento ácido, notou-se que a superficie dentinária encontrava-se com abertura de túbulos, mas a resina estava espalhada, sem penetração ( $\mathrm{Fi}$ gura 3).

Finalmente, nos espécimes do $4^{\circ}$ grupo, os quais receberam um tratamento na superficie da dentina com laser Nd:YAG e procedimento adesivo e restaurador com resina composta, também sem a aplicação do condicionamento ácido, foi possivel observar a não-abertura dos túbulos dentinários. A resina também apresentou-se espalhada sobre a camada dentinária superficial (Figura 4).

Comparativamente, observou-se que a resina somente não se encontrava espalhada na superficie da dentina quando as amostras receberam o tratamento do laser Er:YAG e procedimento adesivo completo. Com o laser Er:YAG, utilizado sem condicionamento ácido, embora tenha havido uma abertura dos túbulos, a resina não conseguiu penetrar na dentina. Nas outras situações com laser Nd:YAG, os aspectos foram praticamente iguais com túbulos vedados portanto sem penetração.

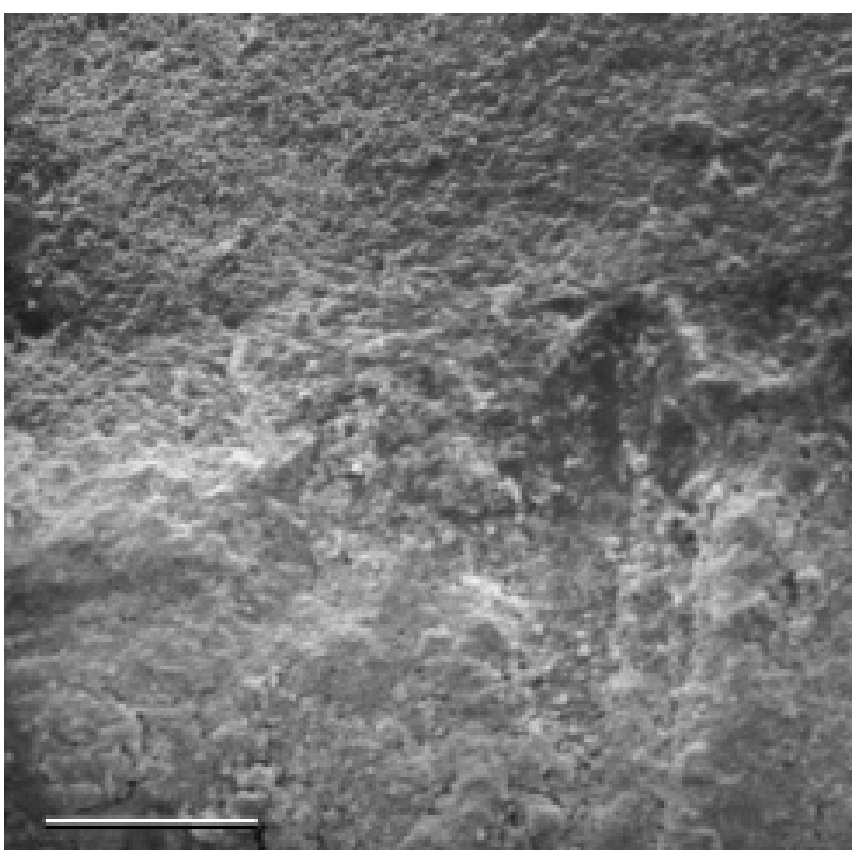

FIGURA 4 - Micrografia de varredura da junção sistema adesivo/resina/dentina tratada com laser Nd:YAG. Observa-se a não-abertura de túbulos (barra de calibração $=50 \mu \mathrm{m})$.

\section{DISCUSSÃO}

A utilização de opções alternativas, como os lasers Er:YAG e Nd:YAG, no tratamento da superficie de esmalte e dentina, para restaurações estéticas adesivas $^{4}$, vem apresentando resultados diversificados. Este fato desperta a necessidade de estudos mais elaborados que nos auxilie na busca de melhores parâmetros para se conseguir esta perfeita união $0^{3,4,6,7,8,13,16,17,18,24,25,26}$. Também se torna evidente que os adesivos devem ser pesquisados para que haja uma maior interação entre a superficie irradiada e o adesivo aplicado.

Em nosso trabalho, variamos a aplicação do condicionamento ácido, para observar, se após esta aplicação, haveria diferenças morfológicas substanciais entre os grupos que receberam o tratamento ácido. A aplicação do condicionamento ácido pode ser desvantajosa, causando o aumento da permeabilidade que leva a uma maior irritabilidade pulpar, podendo ocorrer hidrólise do colágeno com o tempo ${ }^{14,15,20,21}$.

É de extrema importância que se estabeleçam os parâmetros de energia para cada tipo de laser utilizado, nas estruturas determinadas e com finalidades definidas, pois as energias aplicadas em denti- 
ODA, M.; OLIVEIRA, D. C.; LIBERTI, E. A. Avaliação morfológica da união entre adesivo/resina composta e dentina irradiada com laser Er:YAG e laser Nd:YAG: estudo comparativo por microscopia de varredura. Pesqui Odontol Bras, v. 15, n. 4, p. 283-289, out./dez. 2001.

na e esmalte variam ${ }^{16}$, já que o esmalte é altamente mineralizado e a dentina apresenta-se com outras características, tais como a permeabilidade, e estes valores podem modificar as caracteristicas dos tecidos irradiados. Os parâmetros utilizados nesta pesquisa poderiam ser utilizados in vivo. Sabe-se que o laser Nd:YAG é facilmente transmitido através da dentina $^{29}$. Estudos antigos como o de $\mathrm{ZACH}$ et al. ${ }^{28}$ (1965) já demonstravam que o tecido pulpar sadio não é afetado termicamente se o aumento de temperatura for menor que $5^{\circ} \mathrm{C}$. Se o equipamento de laser for utilizado num ajuste correto, e a espessura de dentina remanescente for maior ou igual a $2 \mathrm{~mm}$, também não ocorrerá aumento de temperatura prejudicial à polpa ${ }^{24}$.

Em relação ao laser Er:YAG, utilizamos também uma energia menor, já que com $2 \mathrm{~Hz}, 80 \mathrm{~mJ}$ focado, durante 30 segundos, queríamos evitar que pudesse ocorrer um aumento de temperatura que pudesse ser prejudicial, causando danos à polpa ou tecidos adjacentes, se estivéssemos extrapolando estes parâmetros para trabalhos in vivo ${ }^{27,28}$. Neste estudo, o aumento de temperatura dentro do dente não foi avaliado.

Quando o laser Nd:YAG foi aplicado previamente ao sistema adesivo, independentemente do condicionamento ácido, notamos uma faixa de tecido com ausência de túbulos dentinários abertos, resultado semelhante aos encontrados por TANJI et al. ${ }^{19}$ (1998) e GONÇALVES et al. ${ }^{8}$ (1999). Isto nos leva a acreditar que o laser Nd:YAG, por fundir ou recristalizar a dentina ${ }^{5}$, impede que o adesivo penetre na mesma, não havendo formação de "tags" de resina, e resultando num espalhamento da resina sobre a dentina ${ }^{3,24,25}$. O condicionamento ácido não reverteu este quadro, já que os resultados morfológicos apresentaram-se similares aos do grupo que não utilizou este condicionamento. KAWABATA et al. ${ }^{12}$ (1999) encontraram ainda ao redor dos túbulos dentinários selados várias linhas de fratura de tamanho variável, quando utilizaram energia de 50 a $200 \mathrm{~mJ}$, e $10 \mathrm{~Hz}$. Neste trabalho, com menor energia, 0,6 W, $15 \mathrm{~Hz}, 40 \mathrm{~mJ}$ e tempo de aplicação de 30 segundos, não identificamos tais linhas de fratura.

Nossos resultados em relação ao efeito do laser Er:YAG quando utilizamos também o condicionamento ácido do sistema adesivo foram semelhantes aos encontrados por WIGDOR et $a .^{26}$ (1992); KATAUMI et al. ${ }^{11}$ (1998), VISURI et al. ${ }^{22}$ (1996), ISHIKA-
WA et al. ${ }^{10}$ (1996); TANJI et al. ${ }^{19}$ (1998); e KAWABATA et al. ${ }^{12}$ (1999), com um aspecto morfológico de maior permeabilidade com túbulos abertos, sem apresentar carbonização ou fratura e a dentina também não apresentando "smear layer". HOSSAIN et al. ${ }^{9}$, em 1999, trabalhando com baixa energia, em torno de $100 \mathrm{~mJ}$ e $2 \mathrm{~Hz}$, encontraram túbulos dentinários não visíveis e presença da "smear layer", mas com aumento de energia até $400 \mathrm{~mJ}$ encontraram remoção gradual da "smear layer" e túbulos visiveis. Em nossos resultados, mesmo trabalhando com energia em torno de $80 \mathrm{~mJ}$, e $2 \mathrm{~Hz}$ pudemos ver túbulos dentinários abertos, sem a presença de "smear layer".

Em relação ao condicionamento ácido, quando associado ou não com o laser Er:YAG, tivemos resultados semelhantes, com abertura aparente dos túbulos dentinários. Nesta pesquisa, houve ainda, no grupo que utilizou o laser Er:YAG associado ao condicionamento ácido, penetração da resina. ISHIKAWA et al..$^{10}$, em 1999, observaram que a morfologia da superficie dentinária irradiada e atacada com ácido fosfórico apresentava-se diferente, com a dentina peritubular preservada, provavelmente pelo maior conteúdo mineral desta, em relação à peritubular, e as aberturas dos túbulos ligeiramente alargadas.

Acreditamos que mesmo utilizando parâmetros sugeridos pela literatura ${ }^{13,17}$, há necessidade de mais estudos variando os parâmetros, pois os resultados encontrados segundo nossa metodologia não apresentaram morfologicamente o embricamento mecânico esperado para melhor adaptação da união no sistema adesivo/resina e dentina.

\section{CONCLUSÕES}

Com base nas observações microscópicas, e de acordo com os parâmetros utilizados, concluímos que somente no tratamento na superficie da dentina irradiada com laser Er:YAG e condicionamento ácido ocorreu penetração da resina na dentina. Nos demais tratamentos, houve apenas uma aparente superposição da resina na superficie dentinária, sugerindo que houve oclusão dos túbulos com características de fusão na dentina superficial, quando do uso do laser Nd:YAG, e a necessidade do procedimento adesivo completo, quando com o laser Er:YAG. 
ODA, M.; OLIVEIRA, D. C.; LIBERTI, E. A. Avaliação morfológica da união entre adesivo/resina composta e dentina irradiada com laser Er:YAG e laser Nd:YAG: estudo comparativo por microscopia de varredura. Pesqui Odontol Bras, v. 15, n. 4, p. 283-289, out./dez. 2001.

ODA, M.; OLIVEIRA, D. C.; LIBERTI, E. A. Morphological evaluation of the bonding between adhesive/composite resin and dentin irradiated with Er:YAG and Nd:YAG lasers: comparative study using scanning microscopy. Pesqui Odontol Bras, v. 15, n. 4, p. 283-289, out./dez. 2001.

\begin{abstract}
Since bonding systems were introduced in the restorative procedures carried out with esthetic materials, the treatment of dentin surfaces has been widely studied in order to establish the ideal technique. The application of $37 \%$ phosphoric acid on dentin is still the best known method. However, alternative methods for treating the dentin surface have been discussed in the literature, including the utilization of some kinds of laser irradiation. The purpose of this research was to morphologically evaluate the bond between adhesive materials and the dentin treated with Er:YAG and $\mathrm{Nd}$ :YAG lasers, in a comparative study by means of scanning electron microscopy (SEM). Irradiation either substituted acid etching, or was associated to it. Recently extracted bovine incisors were utilized. They received class $\mathrm{V}$ cavity preparations and were restored with a bonding system and a light-cured composite resin. Meanwhile, some of the teeth underwent irradiation with Er:YAG laser or Nd:YAG laser before the application of the bonding agent and the composite resin. The samples were selected, prepared for SEM and submitted to morphological analysis. Data were registered in photomicrographs. Based on the microscopic observations, we concluded that only in the dentin surfaces submitted to irradiation with Er:YAG laser and to acid conditioning there was penetration of resin into the dentine. With the Nd:YAG laser treatment, there was only visual superposition of resin over the dentin surface, which suggests that there was only occlusion of the tubules, with characteristics of fusion in the superficial dentine.
\end{abstract}

UNITERMS: Lasers; Composite resins.

\section{REFERÊNCIAS BIBLIOGRÁFICAS}

1. BARNES, D. M.; McDONALD, N. J.; THOMPSON, V. P. et al. Microleakage in facial and lingual class 5 composite restorations: a comparison. Oper Dent, v. 19, n. 4, p. 133-137, July/Aug. 1994.

2. BUONOCORE, M. G. A simple method of increasing the adhesion of acrylic filling materials to enamel surfaces. $\mathbf{J}$ Dent Res, v. 34, n. 6, p. 849-853, 1955.

3. COOPER, L. F.; MYERS, M. L.; NELSON, D. G.; MOWERY, A. S. Shear strength of composite bonded to laser-pretreated dentin. J Prosthet Dent, v. 60, n. 1, p. 45-49, 1988.

4. COZEAN, C.; ARCORIA, C. J.; PELAGALLI, J.; POWELL, L. Dentistry for the $21^{\text {st }}$ Century? Erbium:YAG laser for teeth. J Am Dent Assoc, v. 128, n. 8, p. 1080-1087, Aug. 1997.

5. DEDERICH, D. N.; ZAKARIASEN, K. L.; TULIP, J. Scanning electron microscopic analysis of canal wall dentin following neodymium Yttrium-Aluminum-Garnet laser irradiation. J Endod, v. 10, n. 9, p. 428-431, Sept. 1984.

6. EDUARDO, C. P.; CECCHINI, R. C. M.; MARQUES, J. L. L.; MATSUMOTO, K. Scanning electron microscopy study on enamel etching with Nd:YAG laser and phosphoric acid. J Clin Laser Med Surg, v. 13, n. 2, p. 81-85, 1995.

7. EDUARDO, C. P.; OLIVEIRA Jr., W. T.; MYAKI, S. I.; ZEZELL, D. M. Comparative study of the shear bond strength of composite resin to dental enamel conditioned with phosphoric acid or Nd:YAG laser. Rev Odontol Univ São Paulo, v. 11, n. 4, p. 245-248, out./dez. 1997.

8. GONÇALVES, S. E. P.; ARAUJO, M. A. M.; DAMIÃO, A. J. Dentin bond strength: influence of laser irradiation, acid etching, and hypermineralization. J Clin Laser Med Surg, v. 17, n. 2, p. 77-85, 1999.

9. HOSSAIN, M.; NAKAJIMA, M.; YAMADA, T.; TAGAMI, J. Tensile bond strength and SEM evaluation of Er:YAG la- ser-irradiated dentin using dentin adhesive. J Clin Laser Med Surg, v. 17, n. 3, p. 105-109, 1999.

10. ISHIKAWA, H.; AOKI, A.; WATANABE, H. et al. Er:YAG laser: the promising procedure for root caries treatment. In: INTERNATIONAL CONGRESS ON OPTICAL ENGINEERING, 1996. Bellingham : Wash. Proceedings. International Society for Optical Engineering, 1996. p. 131-134.

11. KATAUMI, M.; NAKAJIMA, M.; YAMADA, T.; TAGAMI, J. Tensile bond strength and SEM evaluation of Er:YAG laser-irradiated dentin using dentin adhesive. Dent Mater J, v. 17, n. 2, p. 125-138, 1998.

12. KAWABATA, A.; KAWABATA, H.; YAGASAKI, A. et al. Effects of laser irradiation on dentin - in vitro morphological study following application of various types of lasers. Pediatr Dent J, v. 9, n. 1, p. 83-89, 1999.

13. MATSUMOTO, K. Basic and clinical research on Er:YAG laser in dentistry. In: INTERNATIONAL CONGRESS ON LASERS IN DENTISTRY, 1998, Maui. Proceedings, Maui : International Society of Lasers in Dentistry, 1998. p. 235-236.

14. PASHLEY, D. H.; HORNER, J. A.; BREWER, P. D. Interactions of conditioners on the dentin surface. Oper Dent, p. 137-150, 1992. Suppl. 5.

15. SANO, H.; TAKATSU, T.; CIUCCHI, B. et al. Nanoleakage: leakage within the hybrid layer. Oper Dent, v. 20, n. 1, p. 18-25, 1995.

16. SCHALLER, H. G.; WEIHING, T.; STRUB, J. R. Permeability of dentine after Nd:YAG laser treatement: an in vitro study. J Oral Rehabil, v. 24, n. 4, p. 274-281, Apr. 1997.

17. TAGOMORI, S.; IWASE, T. Ultrastructural change of enamel exposed to a normal pulsed Nd:YAG laser. Caries Res, v. 29, n. 6, p. 513-520, 1995.

18. TANJI, E. Y.; MATSUMOTO, K.; EDUARDO, C. P. Estudo do condicionamento da superficie dentinária com o 
ODA, M.; OLIVEIRA, D. C.; LIBERTI, E. A. Avaliação morfológica da união entre adesivo/resina composta e dentina irradiada com laser Er:YAG e laser Nd:YAG: estudo comparativo por microscopia de varredura. Pesqui Odontol Bras, v. 15, n. 4, p. 283-289, out./dez. 2001.

Er:YAG laser. Apresentado na $13^{a}$ Reunião Científica da SBPqO, Águas de São Pedro, set. 1996.

19. TANJI, E. Y.; SOARES, S. C. G.; EDUARDO, C. P. E. MEV de canais radiculares irradiados com Er:YAG e Nd:YAG laseres. In: $15^{\mathrm{a}}$ REUNIÃO ANUAL DA SOCIEDADE BRASILEIRA DE PESQUISA ODONTOLÓGICA, 1998, Águas de São Pedro. Anais. São Paulo : SBPqO, 1998. p. 47.

20. TAO, L.; PASHLEY, D. H. The relationship between dentin bond strengths and dentin permeability. Dent Mater, v. 5, n. 2, p. 133-139, 1989.

21. TAY, F. R.; GWINNETT, A. J.; PANG, K. M.; WEI, S. H. Resin permeation into acid-conditioned moist, and dry dentin: a paradigm using water-free adhesive primers. J Dent Res, v. 75, n. 4, p. 1034-1044, 1996.

22. VISURI, S. R.; GILBERT, J. L.; WRIGTH, D. D. et al. Shear bond strength of composite bonded to Er:YAG laser-prepared dentin. J Dent Res, v. 75, n. 1, p. 599-605, 1996.

23. YONAGA, K.; KIMURA, Y.; MATSUMOTO, K. Treatment of cervical dentin hypersensitivity by various methods using pulsed Nd:YAG laser. J Clin Laser Med Surg, v. 17, n. 5, p. 205-210, 1999.
24. WHITE, J. M.; GOODIS, H. E.; SETCOS, J. C. et al. Effects of pulsed Nd:YAG laser energy on human teeth: a three-year follow-up study. J Am Dent Assoc, v. 124, n. 6, p. 45-51, July 1993.

25. WHITE, J. M.; GOODIS, H. E.; MARSHALL, S. J. et al. Energy dispersive spectroscopy of laser-modified dentin surfaces. J Dent Res, p. 73, 1994. [Abstract n. 1918]

26. WIGDOR, H.; ASHRAFI, S.; ABT, E. SEM evaluation of $\mathrm{CO}_{2}$, $\mathrm{Nd}$ :YAG and Er:YAG laser irradiation of dentin in vitro. In: INTERNATIONAL CONGRESS ON LASERS IN DENTISTRY, 1992, Salt Lake City. Proceedings, Salt Lake City : International Society of Lasers in Dentistry, 1992. p. 131-132.

27. WIGDOR, H.; ABT, E.; ASHRAFI, S.; WALSH, J. T. The effect of lasers on dental hard tissues. J Am Dent Assoc, v. 124, n. 2, p. 65-70, 1993.

28. ZACH, L.; COHEN, D. H. Pulp response to externally applied heat. Oral Surg Oral Med Oral Pathol, v. 19, p. 515-530, 1965.

29. ZENNYU, K.; INOVE, M.; KONISHI, M. Transmission of Nd:YAG laser through human dentin. J Jpn Soc Laser Dent, v. 7, p. 37-45, 1996.

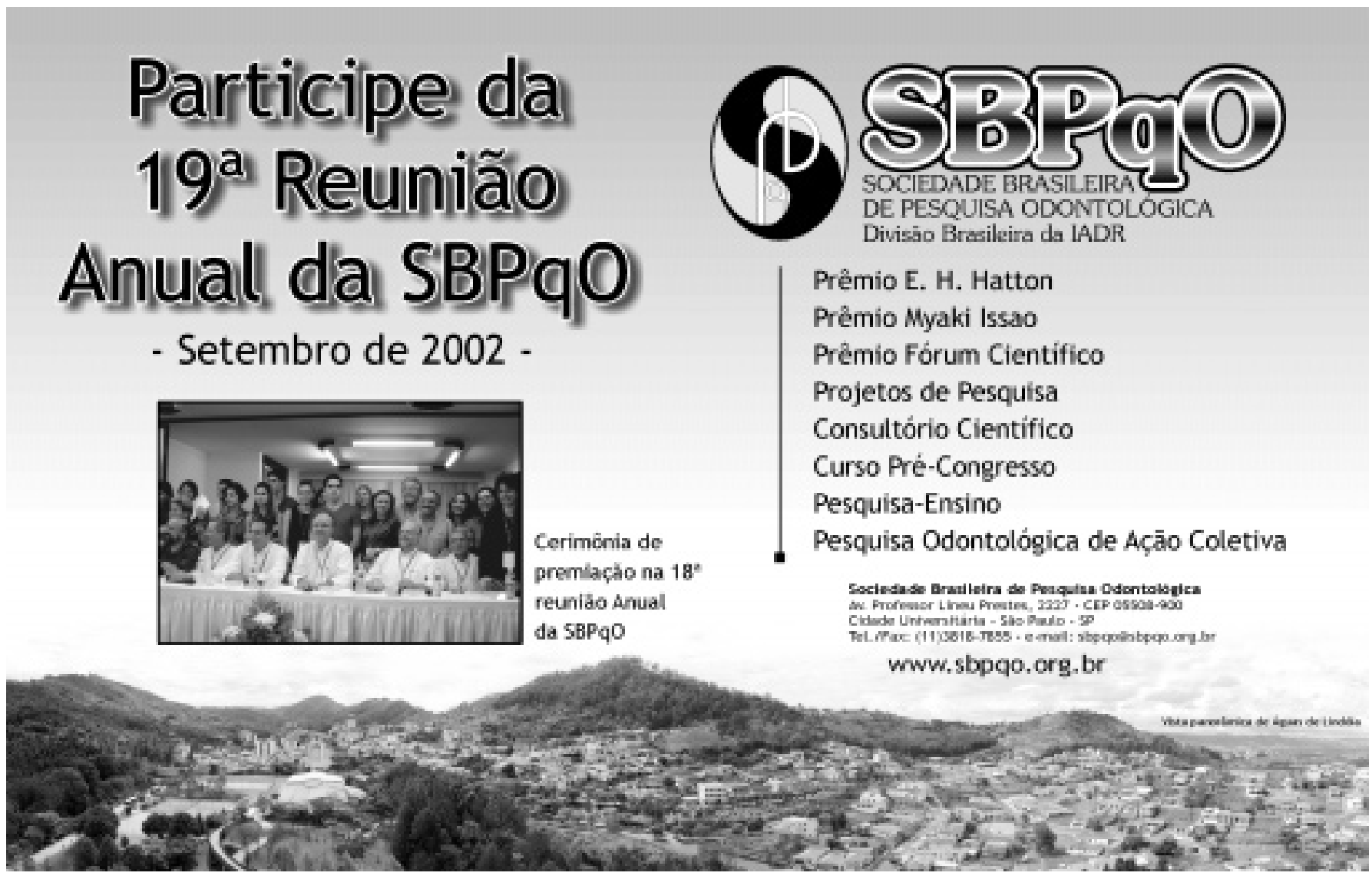

